\title{
AN EXAMPLE IN THE WEIL THEORY OF MEASURABLE GROUPS
}

\author{
ROBERT E. ATALLA
}

\begin{abstract}
According to a theorem of Andre Weil, if a group $G$ possesses an invariant measure which satisfies certain conditions, in particular measurability of the map $(x, y) \rightarrow(x, x y)$ of $G \times G$, then $G$ has a locally bounded Hausdorff topology making $G$ a topological group. We offer a simple counterexample to show the need for the above stated condition.
\end{abstract}

Introduction. A measurable group is a $\sigma$-finite measure space $(G, M, m)$ such that (a) $m$ is not identically zero, (b) $G$ is a group, (c) the $\sigma$-ring $M$ and the measure $m$ are both left invariant, (d) the map $S$ of $G \times G$ given by $S(x, y)=(x, x y)$ is measurability preserving $[1$, p. 257]. The group is called separated if (e) for each $x \in G$ there exists $A \in M$ such that $m(x A \triangle A)>0$, where $\triangle$ is symmetric difference. Weil's theorem states that for a separated measurable group there exists a locally bounded Hausdorff topology on $G$ making it a topological group, with $m(E)>0$ for $E$ open and $m(E)<\infty$ for $E$ bounded [1, p. 273-274].

In this note we give a simple example which illustrates the need for the peculiar measurability hypothesis (d). It will be a system ( $G, M, m)$ such that (a), (b), (c), and (e) hold, while (d) fails. We show that there exists at least one set of the form $\{x: m(x A \triangle A)<\epsilon\}$, with $A \in M$, which is not in the original $\sigma$-ring $M$, contradicting Theorem $C$ on p. 272 of [1].

I am indebted to Professor W. W. Comfort for suggesting in 1966 that I compute this example (in a somewhat different connection).

1. The group. Let $G$ be the set of pairs $(a, t)$, where $a$ is real and $t= \pm 1$, with group operation $(a, t)(b, s)=(a+t b, t s)$. This is isomorphic to the group generated by translations of $R$, plus inversion $I x=-x$. It also has a matrix representation:

$$
(a, t) \sim\left|\begin{array}{cc}
1 & a \\
0 & t
\end{array}\right|
$$

Note that if

Received by the editors December 5, 1969.

AMS Subject Classifications. Primary 2875.

Key Words and Phrases. Topological group, measurable group, Haar measure, invariant measure, Weil topology. 


$$
(b, s) \sim\left|\begin{array}{ll}
1 & b \\
0 & s
\end{array}\right|
$$

then

$$
\left|\begin{array}{ll}
1 & b \\
0 & s
\end{array}\right| \times\left|\begin{array}{cc}
1 & a \\
0 & t
\end{array}\right|=\left|\begin{array}{cc}
1 & a+t b \\
0 & s t
\end{array}\right|
$$

so the product matrix corresponds to $(a, t)(b, s)$ as defined above. This makes it easy to see that $G$ is a group with identity $(0,1)$ and inverse $(a, t)^{-1}=(-t a, t)$.

The $\sigma$-ring $M$ on $G$ is defined as follows: if $A$ is a Borel set in $R$, $A_{0}=A \times\{1\} \cup A \times\{-1\}$. It is easy to check that $M$ is a $\sigma$-ring. We define the measure $m$ by $m\left(A_{0}\right)=l(A)$, where $l$ is Lebesgue measure. $M$ is invariant under left translation, for an easy calculation yields $(b, t) A_{0}=(b+t A)_{0}$. To show $m$ is left invariant, $m\left((b, t) A_{0}\right)=m(b+t A)_{0}$ $=l(b+t A)=l(t A)=l(A)=m\left(A_{0}\right)$ (recall that $\left.t= \pm 1\right)$.

It is obvious that if a Weil topology were to exist, it could not be Hausdorff. For in that topology every set in the neighborhood base is measurable [1, Theorem C, p. 272], while it follows from the definition of $M$ that for any real $a,(a, 1)$ and $(a,-1)$ belong to exactly the same measurable sets.

2. Main results. It is obvious that our system satisfies axioms (a), (b) and (c). That (e) also holds while (d) fails requires proof.

Proposition 1. If $(b, t) \neq(0,1)$, then there exists $A_{0} \in M$ such that $m\left((b, t) A_{0} \triangle A_{0}\right)>0$.

Proof. First, suppose $t=1$. Let $A=[0,1]$. An easy calculation yields $(b, 1)[0,1]_{0}=(b+[0,1])_{0}=[b, 1+b]_{0}$, and so $m\left((b, t) A_{0} \triangle A_{0}\right)$ $=l([b, 1+b] \triangle[0,1])>0$. If $t=-1$ and $b \neq 1$, we again let $A=[0,1]$, getting $(b,-1)[0,1]_{0}=(b-[0,1])_{0}=[-1+b, b]_{0}$, and

$$
m\left((b, t) A_{0} \triangle A_{0}\right)=l([-1+b, b] \triangle[0,1])>0 .
$$

If $t=-1$ and $b=1$, let $A=[-1,1]$. Then

$$
(1,-1)[-1,1]_{0}=(1-[-1,1])_{0}=[0,2]_{0}
$$

and

$$
m\left((b, t) A_{0} \triangle A_{0}\right)=l([0,2] \triangle[-1,1])>0 .
$$

Proposition 2. Axiom (d) for measurable groups fails.

First Proof. Suppose axiom (d) held. Then it follows from Proposition 1 and preceding remarks that $G$ is a separated measurable group, and hence by Weil's Theorem there exists a Hausdorff Weil 
topology for $G$. But this contradicts the remark made at the end of $\S 1$.

Second Proof. Since this is intended to be a simple example, we should not base our main result on the relatively deep theorem of Weil. In this proof we show first that sets in the product measure space $M \times M$ have a certain symmetry property, and then show that this symmetry is not preserved under the map $S$ of axiom (d).

First, if $A$ and $B$ are Borel sets in $R$, then

$$
\begin{aligned}
A_{0} \times B_{0}= & (A \times\{1\} \cup A \times\{-1\}) \times(B \times\{1\} \cup B \times\{-1\}) \\
= & (A \times\{1\} \times B \times\{1\}) \cup(A \times\{1\} \times B \times\{-1\}) \\
& \cup(A \times\{-1\} \times B \times\{1\}) \cup(A \times\{-1\} \times B \times\{-1\}) .
\end{aligned}
$$

Now if $t= \pm 1$ and $s= \pm 1$, let

$$
E(t, s)=\left\{(x, y) \in R \times R:(x, t, y, s) \in A_{0} \times B_{0}\right\} .
$$

Then $E(1,1)=E(1,-1)=E(-1,1)=E(-1,-1)$. It is easy, if messy, to check that this symmetry is preserved under arbitrary unions and intersections, hence pertains to all sets in the product $\sigma$-ring $M I \times M$. Now if $A$ and $B$ are Borel sets in $R$, an easy calculation gives

$$
\begin{aligned}
S\left(A_{0} \times B_{0}\right)= & \{(a,-1, a-b, 1): a \in A, b \in B\} \\
& \cup\{(a, 1, a+b,-1): a \in A, b \in B\} \\
& \cup\{(a,-1, a-b, 1): a \in A, b \in B\} \\
& \cup\{(a, 1, a+b, 1): a \in A, b \in B\} .
\end{aligned}
$$

If $A=B=[0,1]$, then

$$
\begin{aligned}
E(1,1) & =\{(x, x+y): x \in[0,1], y \in[0,1]\}, \\
E(-1,1) & =\{(x, x-y): x \in[0,1], y \in[0,1]\},
\end{aligned}
$$

with $(0,-1) \in E(-1,1)$ and $(0,-1) \notin E(1,1)$.

Proposition 3. There exists $A_{0} \in M$ and $\epsilon>0$ such that

$$
\left\{(a, t): m\left((a, t) A_{0} \triangle A_{0}\right)<\epsilon\right\} \notin M .
$$

Proof. Let $A=[0,1], \epsilon=1 / 3$, and $E$ be the set in question. Then

$$
\begin{aligned}
E= & \left\{(a, 1): m\left((a, 1)[0,1]_{0} \Delta[0,1]_{0}\right)<1 / 3\right\} \\
& \cup\left\{(a,-1): m\left((a,-1)[0,1]_{0} \triangle[0,1]_{0}\right)<1 / 3\right\} \\
= & \{(a, 1): l([a, 1+a] \triangle[0,1])<1 / 3\} \\
& \cup\{(a,-1): l([-1+a, a] \triangle[0,1])<1 / 3\} \\
= & \{(a, 1): a \in(-1 / 3,1 / 3)\} \cup\{(a,-1): a \in(2 / 3,4 / 3)\} .
\end{aligned}
$$


Since $M$ consists exclusively of "symmetric" sets of the form $A \times\{1\} \cup A \times\{-1\}$, it follows that $E \notin M$.

\section{REFERENCES}

1. P. R. Halmos, Measure theory, Van Nostrand, Princeton, N. J., 1950. MR 11, 504.

Ohio University, Athens, Ohio 45701 